\section{Stimulus tape generator: A package to present analog recordings according to user specifications}

\section{GILBERT SOOHOO and ALLEN A. MONTGOMERY Army Audiology and Speech Center Walter Reed Army Medical Center, Washington, $D C$}

This computer software package is useful to the person who has a series of tape recordings of brief stimuli and who desires to prepare a new tape recording of the stimuli. The stimuli on the new recording may be reordered, may have different temporal spacing between them, and/or may have altered recording levels. In general, the user prepares a digitized set of items through analog-to-digital conversion (ADC) of analogy recordings (usually speech). The pool of items may then be adjusted for RMS amplitude (in decibels) under several options if necessary, and presented for rerecording through digital-to-analog conversion (DAC) under a variety of options controlling ordering and spacing. Specific applications would include: preparing speech audiometry tapes with exact spacing between items; presenting items for paired comparison with a specified pause between paired members and a longer specified pause between pairs; $A B X$ presentations; and pairedassociate learning tasks.

\section{Environment}

The program package was developed on a $128 \mathrm{~K}$ PDP-11/34 equipped with RK05 and RK07 disks, ADC/DAC, Schmitt trigger, and LPSKW real-time clock. The operating system was RT11 V3B, and processor languages were FORTRAN and MACRO 11. The analog equipment used in implementing this package include an audiotape recorder, filter, amplifier, and speaker.

\section{Stimulus Tape Generator}

The programs are divided into three groups according to function.

(1) Preeditor. STGPRE samples user-supplied analog stimuli via $\mathrm{ADC}$ at $10 \mathrm{kHz}$ and stores those samples on disk. Maximum sampling duration is $1.5 \mathrm{sec}$. The sampling speed can be altered in the source code; but given the maximum of 15,360 samples, the sampling duration

Software development received financial support from the Department of Clinical Investigation, Walter Reed Army Medical Center, under Work Unit 2536. The opinions or assertions contained herein are the private views of the authors and are not to be construed as official or as reflecting the views of the Department of the Army or the Department of Defense. Requests for reprints should be sent to Gilbert SooHoo, Army Audiology and Speech Center, Walter Reed Army Medical Center, Washington, DC 20307. would decrease accordingly. Longer samples are possible if there is sufficient memory. The newly sampled analog stimulus may be presented aurally via DAC coupled to a speaker for user assessment. At this point, the user may specify which part of the sample he desires to save on disk. Typically, dead spaces found both before and after the desired sample are edited out, at this stage, thereby conserving disk storage. The user need not be concerned with the files' internal structure. He must, however, ensure sufficient disk space for all his stimulus files. Disk space requirements can be computed by the following formula: $D_{\text {space }}=\left(T_{\text {sample }} \cdot R_{\text {sam ple }}\right) / 256$. Thus, disk space $\left(D_{\text {space }}\right)$ is in units of disk blocks (unformatted binary files in Files-11 format). And $T_{\text {sample }}$ and $R_{\text {sample }}$ are the sampling duration (in seconds) and sampling rate, respectively. The maximum duration of $1.5 \mathrm{sec}$ at $10 \mathrm{kHz}$ would then result in a 60 -block file. A maximum of 79 such files can be stored on an RK05 disk.

(2) Editor. STGEDI edits stimulus disk files that were created by the preeditor STGPRE. Level adjustments may be made: The signal amplitude of several files may be averaged across files or normalized to that of a selected file. Presentation order of the analog stimuli can be user specified or random (random sequences are permitted for single or paired stimuli, but not for triads). The minimum pause between stimuli is $.5 \mathrm{sec}$, and the maximum is $49.5 \mathrm{sec}$. As many as 200 stimuli (i.e., 200 single stimuli, 100 paired stimuli, or 66 triad stimuli) can be presented in a series. In regard to pairs and triads, the user needs to specify the combinations desired. For pairs, however, he may specify all possible pairs with at most 10 distinct stimuli.

(3) Posteditor. STGPOS presents the analog stimuli in the desired sequence via DAC. The user has the option, in our setting, of channeling the output to either a speaker or an audiotape recorder (or both).

\section{Availability}

A listing of the source code and a user's guide are available at no cost. Interested persons may send a formatted RK05 cartridge for a copy of the source and executable modules.

\section{Portability}

The package should run on any Digital computer system with at least $64 \mathrm{~K}$ of memory under RT-11 V3 or above. The computer system must be equipped with a Laboratory Peripheral System (LPS) and AA-11K DAC; otherwise, the device register memory specifications need to be modified to accommodate different peripherals.

(Manuscript accepted for publication March 23, 1984.) 